\title{
IRDiRC: 1000 new rare diseases treatments by 2027, identifying and bringing forward strategic actions
}

\author{
Virginie Hivert ${ }^{1,2}$, Anneliene H. Jonker ${ }^{1,3}$, Daniel O'Connor ${ }^{1,4}$, Diego Ardigo ${ }^{1,5}$ \\ ${ }^{1}$ International Rare Diseases Research Consortium (IRDiRC), Paris 75013, France. \\ ${ }^{2}$ EURORDIS - Rare Diseases Europe, Paris 75014, France. \\ ${ }^{3}$ TechMed Centre, University of Twente, Enschede $7522 \mathrm{NH}$, The Netherlands. \\ ${ }^{4}$ Medicines and Healthcare product Regulatory Agency, London E14 4PU, UK. \\ ${ }^{5}$ Chiesi Farmaceutici S.p.A., Parma 43122, Italy.
}

\begin{abstract}
Correspondence to: Dr. Anneliene H. Jonker, TechMed Centre, University of Twente, Hallenweg 5, Enschede 7522 NH, The Netherlands. E-mail: a.h.jonker@utwente.nl
\end{abstract}

How to cite this article: Hivert V, Jonker AH, O'Connor D, Ardigo D. IRDiRC: 1000 new rare diseases treatments by 2027,
identifying and bringing forward strategic actions. Rare Dis Orphan Drugs J 2021;1:3. https://dx.doi.org/10.20517/rdodj.2021.02

Received: 16 Jul 2021 First Decision: 24 Aug 2021 Revised: 13 Sep 2021 Accepted: 19 Oct 2021 Published: 1 Nov 2021

Academic Editor: Daniel Scherman Copy Editor: Xi-Jun Chen Production Editor: Xi-Jun Chen

\begin{abstract}
In 2017, the International Rare Diseases Research Consortium (IRDiRC) set out ambitious goals, one of which specifically aimed to stimulate the development and approval of 1000 new therapies for rare diseases by 2027 . This goal was part of IRDiRC's concerted efforts to foster research and provide better diagnostics and care options for the estimated 400 million patients suffering from the more than 6000 rare diseases (RD) worldwide. Lack of therapeutic options for rare disease patients is an urgent issue. Treatments are estimated to be available for less than $6 \%$ of RD conditions, and fewer than 50 new therapies per year are approved by regulatory agencies worldwide, leaving a major discrepancy between patient needs and therapeutic solutions. This paper describes the recent key steps the IRDiRC Therapies Scientific Committee (TSC) has taken to support the future approval of 1000 new therapies, namely Step 1 (conducting a gap analysis of the rare diseases drug development landscape) and Step 2 (developing strategic themes to advance IRDiRC Goal 2 and act upon them). The IRDiRC TSC created a multi-stakeholder group to run a gap analysis of the RD drug development field. The analysis identified four main priority needs: (1) the definition of a new master plan for RD medicines suitable for all developers (large and small pharmaceutical companies, academics, and not-for-profit organizations) incorporating stakeholders' perspectives and best practices in the field to increase efficiency in the development and registration of innovative drugs and generate more value for patients and the healthcare system; (2) the elicitation of a research framework and business model for repurposing of existing drugs for RD indications to enact a quantum enlargement of the existing
\end{abstract}


therapeutic armamentarium; (3) the definition of standards and practices for data collection in healthcare practice and their implementation in drug development to provide real-world evidence; and (4) the re-focusing of the current international RD research agenda pushing for concentrated research efforts and funding in support of the development of future treatments. In addition to identifying where efforts should be put, the TSC has concretely contributed to advance the IRDiRC goal by creating tools (e.g., the Orphan Drug Development Guidebook) and recommendations and making them available to the whole RD community. However, much remains to be done, and the TSC has refined its approach to incorporate progress made and reflect on new challenges.

Keywords: IRDiRC, orphan drugs, orphan medicinal products, medicines development, gap analysis, rare diseases, rare diseases therapies, rare diseases treatments

\section{INTRODUCTION}

Rare diseases (RD) are conditions that have been historically neglected, because of both their complexity and the rarity of their clinical presentations. There are barriers to establishing a timely diagnosis of these patients and unpredictability and challenges when it comes to therapeutic development. Following on from $\mathrm{RD}$ patient advocacy groups actions and the promulgation of specific supportive orphan drug regulations aiming at attracting investment in the field, the United States NIH and the European Commission, joined by a significant number of public and private research funders, came together a decade ago to create an International Consortium to foster RD research, The International Rare Diseases Research Consortium (IRDiRC).

IRDiRC, officially launched in 2011, was originally conceived with two main goals: (1) contribute to the development of 200 new therapies; and (2) provide the means to diagnose most rare diseases by 2020 . The goal to deliver 200 new therapies was achieved in early $2017^{[1]}$. Despite this progress, published literature suggests that no more than $6 \%$ of the approximate $6000 \mathrm{RD}$ affecting an estimated 400 million patients worldwide ${ }^{[2,3]}$ have an approved treatment. Capitalizing on the momentum of the progress of the past years, IRDiRC devised a new set of global rare disease goals for the decade 2017-2027 $7^{[4]}$. Goal 2, the focus of this paper, is "1000 new therapies for rare diseases will be approved, the majority of which will focus on diseases without approved options" (other goals include Goal 1 "All patients coming to medical attention with a suspected rare disease will be diagnosed within one year if their disorder is known in the medical literature; all currently undiagnosable individuals will enter a globally coordinated diagnostic and research pipeline" and Goal 3 "Methodologies will be developed to assess the impact of diagnoses and therapies on rare disease patients").

The definition of Goal 2 was based on the tracking of the evolution of the RDs drug development field and its emerging trends during the previous years.

Within IRDiRC, the Therapies Scientific Committee (TSC) is a multi-stakeholder group of experts in medical research and drug development ${ }^{[6]}$ devoted to driving IRDiRC's therapeutic development agenda. In accordance with our mandate, we tasked ourselves as TSC members to come up with an ambitious strategy to help address Goal 2. The purpose of this paper is to describe the steps we followed over the last three years, starting with a gap analysis and moving to the development of tools and recommendations that can advance the goal. Finally, we describe elements of action that will bring this work to full fruition by 2027.

The development of medicinal products for RDs is a challenge on many fronts, scientifically, time- and resource-wise, and financially. To successfully bring a drug onto the market, developers need to overcome 
various problems: lack of a body of knowledge on the disease and its natural history; the heterogeneity of its clinical presentation; low, sometimes very low, prevalence which makes the identification of patients difficult and impacts the clinical trial design and methodology; and many diseases affect the pediatric population requiring special considerations, among others. To help overcome some of these obstacles and foster the development of products to treat RDs, different regulatory and economic incentives were set out around the globe $e^{[7,8]}$. Until now, this has delivered over 500 drugs that have reached the market globally ${ }^{[9,10]}$.

However, it is estimated that fewer than 50 new therapies per year are approved by regulatory agencies worldwide, leaving a major gap between patient needs and therapeutic solutions. At the current pace of drug development, i.e., an average of 50 new therapies developed per year, it would take over 100 years to get a single treatment for all 6000 conditions, notwithstanding the fact that addressing patient needs is not only a question of the number of drugs but also to what extent these drugs address the unmet medical need. Based on these reflections, one of the first statements we were able to make is that there is a need for a quantum change in the speed and way of delivery of the present drug development model and ecosystem for rare diseases.

\section{Step 1: conducting a gap analysis of the rare diseases drug development landscape}

To determine which actions should be taken to help achieve IRDiRC Goal 2, TSC members performed a strategic gap analysis [Figure 1A]. This analysis started with a structured questionnaire distributed to each of the fourteen individual TSC members, from different stakeholder groups, allowing the gathering of expert opinion from academic and clinical researchers, regulators, company representatives, health technology assessment representatives, and representatives from patient organizations. The questionnaire provided a framework for systematically exploring the steps along the drug development process and qualitative analysis of the feedback for the creation of a list of items representing the identified gaps (classified from a technical, legal/regulatory, political, societal, or other standpoint). A scoring exercise was then performed to establish a list of priority gaps; the ranking took into account the extent of the (positive) expected impact when the gap is overcome as well as to what extent the gap can be tackled by IRDiRC and its members.

The second set of open questions was targeting what actions should be undertaken to work towards overcoming these gaps. The aggregated list of proposed actions was refined through a brainstorming exercise with the group, which delivered a consolidated list of priority actions.

The third objective was to build a stakeholder map and apply a stakeholder behavior SWOT system to identify which categories of stakeholders were the most likely to be interested and able to resolve the identified gaps combined with IRDiRC's capacity to leverage their participation (interest $v$ s. influence matrix).

The combination of these three outcomes, namely list of priority gaps, list of priority actions, and SWOT analysis, was cross-checked with past, ongoing, and planned IRDiRC activities - inside and outside the TSC - to avoid overlaps. This laid the foundation of the TSC future strategic action plan and separated it into strategic imperatives, strategic tools, and targeted stakeholder engagement [Figure 1B].

Four clusters of items came out very strongly from the gap analysis highlighting: (1) the complexity and challenges of the development model as a whole; (2) the under-use of the repurposing approaches; (3) the importance of data collection and data management in the development of the medicines and the evolution of the field [e.g., FAIR (Findable, Accessible, Interoperable and Reusable) data, real world evidence, 

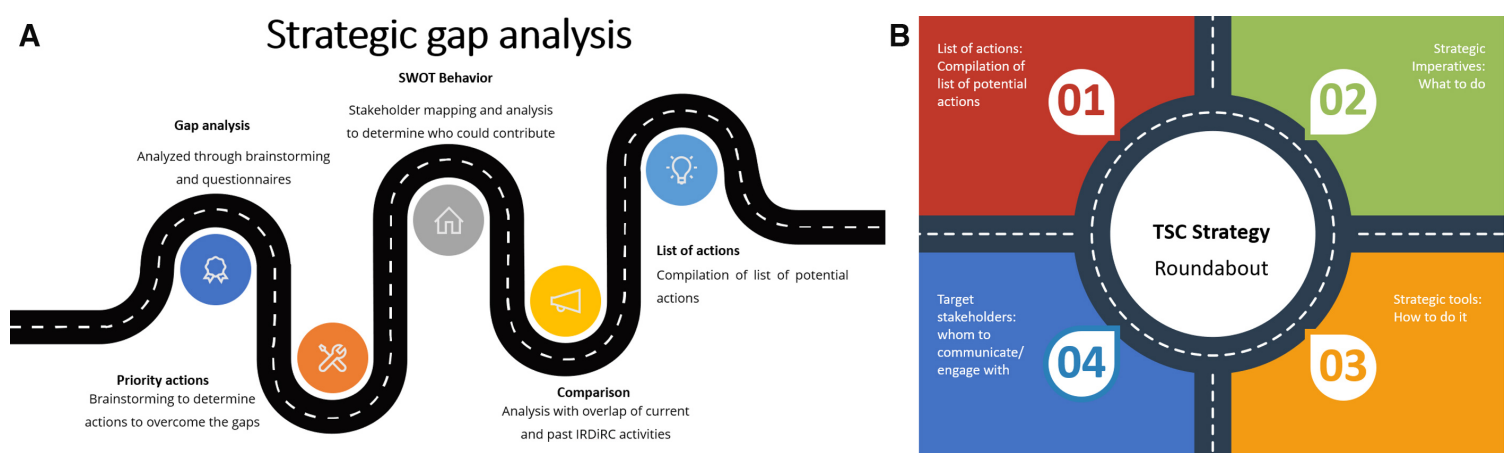

Figure 1. The three workstreams of strategic gap analysis, in which TSC members systematically explored the steps along the drug development process, identifying gaps and actions to be taken and who to involve in the process (A) and the combination of these assets with a direct suggestion list laying out the three pillars of the TSC strategy (B). These figures are based on Showeet.com template. TSC: Therapies Scientific Committee; IRDiRC: International Rare Diseases Research Consortium.

decentralized trials, artificial intelligence, etc.]; and (4) the necessity of a targeted RD research agenda.

The next step of our approach was to turn imperatives into actions and propose four strategic themes to the IRDiRC. For each of them, we reframed the strategic imperatives and identified the strategic tools to be used, the key stakeholders to consult, the actions to be taken, and the foreseen deliverables.

\section{Step 2: developing the four strategic themes to advance IRDiRC Goal 2}

Following the gap analysis described in Step 1, four themes were identified as requiring further action. In the next part of the paper, we go through each of them and highlight which actions have been taken. Beyond these four main themes clustered around the concept of "development model", "drug repurposing", "data collection", and "research agenda", some other aspects have surfaced. These represent more transversal issues to be tacked, such as increase knowledge about RDs in society; multi-stakeholder education programs; aspects related to access to therapies including harmonization of regulatory and HTA requirements across regions; price trends and access to orphan drug; and expansion of availability of drugs in low-income countries. Although they do not represent the focus of this paper as they are not currently being actively taken forward by the TSC, they are certainly highly relevant for the RD field. Some of these are or have been tackled in other IRDiRC groups ${ }^{[11]}$, with assistance of the TSC where necessary.

\section{Strategic Theme 1: support the definition of a new master plan for the development and registration of} innovative drugs specific to rare diseases

This theme built upon two previous areas tackled by the TSC members through task forces on "patientcentered outcome measures in rare diseases" and "small population clinical trials". These resulted in a set of published recommendations ${ }^{[12,13]}$. However, although these two task forces have brought clarification on the concept and practices in the field, there remained a need for an integrated approach that could further support and guide drug developers. With the mindset that each therapeutic development plan has to start from identifying the patient's need while also ensuring systematic engagement with patients all along the drug development pathway ${ }^{[14]}$, we spearheaded the development of the IRDiRC Orphan Drug Development Guidebook (ODDG) $)^{[15]}$.

This Guidebook is based on the finding that a lot of "tools" (e.g., regulatory orphan drug designation), initiatives, and resources are at the disposal of the developers in the RD field, but it is not always easy to identify which of these elements are available and which are to be used when and how. To improve the transparency and efficiency of the development model, the ODDG provides an innovative and unique 
resource to expedite this process by systematically organizing the tools available in Europe, the United States, and Japan. Each of these elements is positioned in a structured framework representing the different milestones of an RD medicine lifecycle, hence providing a map to navigate the therapeutic development ecosystem $^{[16-18]}$.

All the elements of the ODDG have been compiled on a user-friendly website ${ }^{[19]}$. Development cases have been created to illustrate the different types of possible development, i.e., for a traditional or highly innovative technology and whether it concerns a "sufficiently well-understood disease" or "less understood" rare disease, which is quite often the case.

All the materials and recommendations produced and constituting this ODDG are freely available on the IRDiRC website, and an agreement has been made with the European Joint Programme on Rare Diseases $(\mathrm{EJP}-\mathrm{RD})^{[20]}$ to ensure its sustainability. By ensuring the dissemination of this Guidebook ${ }^{[1,2,21]}$ and the uptake of its content (to be updated over time) by user organizations, we aim at enhancing the identification of the available tools so that delays in development timelines can be avoided, risks and costs reduced, and regulatory acceptability and patient access to treatment improved. This work also illustrated some areas where additional efforts should be put, for example in the context of repurposing approaches, as discussed below.

Strategic Theme 2: support the definition of a research framework and business model for repurposing existing drugs specific to RDs

The repurposing of existing drugs into rare indications has allowed for the placing onto the market of new treatment opportunities for patients with rare diseases. Besides regulatory dispositions - such as orphan drug designation and Paediatric Use Marketing Authorisation in Europe - repurposing approaches have been supported in recent years by dedicated funding calls from for example the United States National Center for Advancing Translational Sciences and E-Rare European Research Area (ERA-NET).

During 2016-2017, the TSC led the Data Mining and Repurposing (DMR) Task Force which addressed the issue of identification of opportunities for repurposing ${ }^{[22]}$. In reviewing past successes of data mining for drug repurposing and planning for future biomedical research capacity, the DMR Task Force identified four strategic infrastructure investment areas to focus on to accelerate rare disease research productivity and drug development: (1) improving the capture and sharing of self-reported patient data; (2) better integration of existing research data; (3) increasing experimental testing capacity; and (4) sharing of rare disease research and development expertise. Additionally, the DMR Task Force also recommended a number of strategies to increase data mining and repurposing opportunities for rare diseases research as well as the development of individualized and precision medicine strategies.

The biggest issue remains, however, related to how to attract investments and make these opportunities move forward into the development pathway with the goal to make treatments available to RD patients.

Based on this finding and building on initiatives, such as the Safe and Timely Access to Medicines for Patients and the National Institutes of Health Therapeutics for Rare and Neglected Diseases program ${ }^{[22-24]}$, the TSC has created two workstreams: (1) the elicitation of sustainable economic models suitable for repurposing approaches, i.e., what are the types of business models which could close the gap between attractiveness for developers and access to therapies for patients; and (2) the identification and creation of tools, incentives, and initiatives for data mining and repurposing to create a Drug Repurposing Guidebook, following the same methodology as the ODDG. 


\section{Strategic Theme 3: data collection in healthcare practice}

The third theme identified was related to the need for developing knowledge and understanding of rare diseases, for example through natural history studies, and using the full capacity of research and clinical networks. Hence, the group was calling for support in the creation of common data standards and rules for data collection, data sharing, and use and reuse of data, in particular in healthcare practice for real-world evidence generation in RDs. These aspects are considered crucial to further the ambition of Goal 2, cross covering multiple areas and disciplines. Therefore, it was considered that this theme might be strategically addressed from the IRDiRC as a whole.

\section{Strategic Theme 4: international research agenda for $R D s$}

The last strategic theme was highlighting the need for the reframing of the current international research agenda for RDs with a more coordinated approach within and between countries and worldwide, specifically identifying key criteria that would make public and private research in rare diseases more active and efficient across borders. This is clearly the very purpose of IRDiRC, and thus, as with the previous theme, it was felt that this overarching theme would benefit from a cross-consortium approach.

\section{CONCLUSIONS}

Across the 6000 rare diseases identified, we observe a spectrum that goes from diseases benefitting from several treatment options (although not necessarily curative ones) to diseases with one medicinal product with a Market Authorization, followed by diseases with product(s) in development, or research ongoing, to diseases that remain completely unresearched. The RD Community is calling for equity, and there is a need for a quantum change in the speed and way of delivery of the present drug development model.

To support the goal of 1000 new therapies by 2027 (IRDiRC Goal 2), the IRDiRC TSC conducted a series of activities. Step 1 involved conducting a gap analysis of the rare diseases drug development landscape and Step 2 was developing strategic themes to advance Goal 2. The identified strategic themes are:

- Strategic Theme 1: support the definition of a new master plan for the development and registration of innovative drugs specific to rare diseases.

- Strategic Theme 2: support the definition of a research framework and business model for repurposing existing drugs specific to RDs.

- Strategic Theme 3: data collection in healthcare practice.

- Strategic Theme 4: international research agenda for RDs.

Of these four strategic themes, the TSC has to date proactively advanced two of them (Strategic Themes 1 and 2). For Strategic Theme 1, we developed with colleagues a new roadmap for the development and registration of drugs specific to rare diseases, leading to the publication of the ODDG that provides tools and recommendations for developers to navigate through the $\mathrm{RD}$ development ecosystem. Strategic Theme 2 is currently ongoing, but we aim to unlock the potential of drug repurposing for bringing therapeutic solutions to RD patients, through a dedicated workstream on Elicitation of sustainable economic models suitable for repurposing approaches (manuscript in preparation) and the development of the Drug Repurposing Guidebook (building on the success of the ODDG). 
Additional efforts and measures need to be taken, including at the policy level. In particular, future taskforces should look at how to unlock the potential of currently disregarded - and often ultra-rare diseases. It is hoped that these current and future strategic activities of the TSC will help contribute to the progress being made in the RD field worldwide and the successful completion of the IRDiRC Goal 2.

\section{DECLARATIONS}

\section{Acknowledgement}

Members and contributors of the IRDiRC Therapies Scientific Committee are Annemieke Aartsma-Rus (Leiden University Medical Center, The Netherlands), Diego Ardigo (Chiesi Farmaceutici S.p.A., Italy), Seng Cheng (Pfizer, United States), Robin Conwit (National Institute of Health, United States), Michela Gabaldo (Fondazione Telethon, Italy), Virginie Hivert (EURORDIS - Rare Diseases Europe, France), Sangeeta Jethwa (Roche, Switzerland), Anneliene Jonker (University of Twente, The Netherlands), Yann Le Cam (EURORDIS-Rare Diseases Europe, France), Akifumi Matsuyama (Osaka, Japan), Janet Maynard (US FDA, USA), Thomas Morel (KU Leuven/ UCB, Belgium), Daniel O'Connor (Medicines and Healthcare products Regulatory Agency, United Kingdom), Karin Rademakers (Utrecht, The Netherlands), Ken Sakushima (Japan Pharmaceutical and Medical Devices Agency, Japan) and Maurizio Scarpa (MetabERN and University of Padova, Italy).

\section{Authors' contributions}

Drafted and revised the manuscript and approved the final draft: Hivert V, Jonker AH, O'Connor D, Ardigo D

They are listed in the order representing their contribution to the writing of the manuscript.

\section{Availability of data and materials}

The data supporting the findings of this study are available within the article.

\section{Financial support and sponsorship}

The Therapies Scientific Committee and the IRDiRC Scientific Secretariat were supported by the European FP7 Contract "Support-IRDiRC" ( $\mathrm{N}^{\circ}$ 305207) and are currently supported by the European H2020 Contract “EJP RD” ( $N^{\circ}$ 825575). The authors haven't received any specific funding, nor their respective organisations.

\section{Conflicts of interest}

The views expressed in this publication are the sole responsibility of the authors and do not necessarily reflect the views of their affiliated organizations. All authors declared that there are no conflicts of interest.

\section{Ethical approval and consent to participate}

Not applicable.

\section{Consent for publication}

All TSC members have consented to the roadmap and its information, as presented in this paper, being published.

\section{Copyright}

(c) The Author(s) 2021.

\section{REFERENCES}

1. Dawkins HJS, Draghia-Akli R, Lasko P, et al; International Rare Diseases Research Consortium (IRDiRC). Progress in rare diseases research 2010-2016: an IRDiRC perspective. Clin Transl Sci 2018;11:11-20. DOI PubMed PMC

2. World Health Organization. Rare diseases - background paper. Available from: https://www.who.int/medicines/areas/priority_medicines/Ch6_19Rare.pdf [Last accessed on 20 Oct 2021]. 
3. Orphanet. Orphanet report series - prevalence and incidence of rare diseases. Available from: https://www.orpha.net/orphacom/cahiers/docs/GB/Prevalence_of_rare_diseases_by_alphabetical_list.pdf [Last accessed on 20 Oct 2021].

4. Vision \& Goals. Available from: https://irdirc.org/about-us/vision-goals/ [Last accessed on 20 Oct 2021].

5. Austin CP, Cutillo CM, Lau LPL, et al; International Rare Diseases Research Consortium (IRDiRC). Future of rare diseases research 2017-2027: an IRDiRC perspective. Clin Transl Sci 2018;11:21-7. DOI PubMed PMC

6. IRDiRC. Therapies Scientific Committee (TSC). Available from: https://irdirc.org/about-us/people-organisation/scientificcommittees/tsc/ [Last accessed on 20 Oct 2021].

7. Orphan Drug Act - relevant excerpts. Available from: https://www.fda.gov/industry/designating-orphan-product-drugs-and-biologicalproducts/orphan-drug-act-relevant-excerpts [Last accessed on 20 Oct 2021].

8. European Commission. Regulation No 141/2000 of the European Parliament and of the Council on orphan medical products. Available from: https://ec.europa.eu/health/sites/health/files/files/eudralex/vol-1/reg_2000_141_cons-2009-07/reg_2000_141_cons-200907_en.pdf [Last accessed on 20 Oct 2021].

9. Boycott KM, Ardigó D. Addressing challenges in the diagnosis and treatment of rare genetic diseases. Nat Rev Drug Discov 2018;17:151-2. DOI PubMed

10. RARE Facts. Global Genes. Available from: https://globalgenes.org/rare-disease-facts/ [Last accessed on 20 Oct 2021].

11. Gahl WA, Wong-Rieger D, Hivert V, Yang R, Zanello G, Groft S. Essential list of medicinal products for rare diseases: recommendations from the IRDiRC Rare Disease Treatment Access Working Group. Orphanet J Rare Dis 2021;16:308. DOI PubMed PMC

12. Day S, Jonker AH, Lau LPL, et al. Recommendations for the design of small population clinical trials. Orphanet $J$ Rare Dis 2018;13:195. DOI PubMed PMC

13. Morel T, Cano SJ. Measuring what matters to rare disease patients - reflections on the work by the IRDiRC taskforce on patientcentered outcome measures. Orphanet J Rare Dis 2017;12:171. DOI PubMed PMC

14. The innovation imperative: drug development. EIU Parexel. Available from: https://druginnovation.eiu.com/ [Last accessed on 20 Oct 2021].

15. Hechtelt Jonker A, Hivert V, Gabaldo M, et al. Boosting delivery of rare disease therapies: the IRDiRC Orphan Drug Development Guidebook. Nat Rev Drug Discov 2020;19:495-6. DOI PubMed

16. Malinowski KP, Kawalec P, Trabka W, Sowada C, Pilc A. Reimbursement of orphan drugs in Europe in relation to the type of authorization by the European Medicines Agency and the Decision Making based on Health Technology Assessment. Front Pharmacol 2018;9:1263. DOI PubMed PMC

17. Nicod E, Annemans L, Bucsics A, Lee A, Upadhyaya S, Facey K. HTA programme response to the challenges of dealing with orphan medicinal products: process evaluation in selected European countries. Health Policy 2019;123:140-51. DOI PubMed

18. Precision Medicine. Available from: https://www.fda.gov/medical-devices/vitro-diagnostics/precision-medicine [Last accessed on 20 Oct 2021].

19. IRDiRC Orphan Drug Development Guide. Available from: https://orphandrugguide.org/ [Last accessed on 20 Oct 2021].

20. EJP RD - European Joint Programme on rare diseases. Available from: https://www.ejprarediseases.org/ [Last accessed on 20 Oct 2021].

21. Orphan Drug Development Guidebook Materials. Available from: https://irdirc.org/orphan-drug-development-guidebook-materials/ [Last accessed on 20 Oct 2021].

22. Southall NT, Natarajan M, Lau LPL, et al; IRDiRC Data Mining and Repurposing Task Force. The use or generation of biomedical data and existing medicines to discover and establish new treatments for patients with rare diseases - recommendations of the IRDiRC Data Mining and Repurposing Task Force. Orphanet J Rare Dis 2019;14:225. DOI PubMed PMC

23. DAUE R. Commission Expert Group on safe and timely access to medicines for patients ("STAMP"). Available from: https://ec.europa.eu/health/documents/pharmaceutical-committee/stamp_en [Last accessed on 20 Oct 2021].

24. Drug repurposing screening for rare and neglected diseases. Available from: https://ncats.nih.gov/trnd/projects/drug-repurposing [Last accessed on 20 Oct 2021]. 